\title{
Angiotensin-converting enzyme 2 (ACE2) proteins of different bat species confer variable susceptibility to SARS-CoV entry
}

\author{
Yuxuan Hou $\cdot$ Cheng Peng $\cdot$ Meng Yu $\cdot$ Yan Li $\cdot$ \\ Zhenggang Han $\cdot$ Fang Li $\cdot$ Lin-Fa Wang • \\ Zhengli Shi
}

Received: 21 April 2010/Accepted: 12 June 2010/Published online: 22 June 2010

(C) Springer-Verlag 2010

\begin{abstract}
The discovery of SARS-like coronavirus in bats suggests that bats could be the natural reservoir of SARS$\mathrm{CoV}$. However, previous studies indicated the angiotensinconverting enzyme 2 (ACE2) protein, a known SARS-CoV receptor, from a horseshoe bat was unable to act as a functional receptor for SARS-CoV. Here, we extended our previous study to ACE2 molecules from seven additional bat species and tested their interactions with human SARS-CoV spike protein using both HIV-based pseudotype and live SARS-CoV infection assays. The results show that ACE2s of Myotis daubentoni and Rhinolophus sinicus support viral entry mediated by the SARS-CoV S protein, albeit with different efficiency in comparison to that of the human ACE2. Further, the alteration of several key residues either decreased or enhanced bat ACE2 receptor efficiency, as predicted from a structural modeling study of the different
\end{abstract}

Electronic supplementary material The online version of this article (doi:10.1007/s00705-010-0729-6) contains supplementary material, which is available to authorized users.

Y. Hou $\cdot$ C. Peng $\cdot$ Y. Li $\cdot$ Z. Han $\cdot$ Z. Shi $(\bowtie)$

State Key Laboratory of Virology, Wuhan Institute of Virology, Chinese Academy of Sciences (CAS), Wuhan 430071, Hubei, China

e-mail: zlshi@wh.iov.cn

M. Yu · L.-F. Wang $(\bowtie)$

Australian Animal Health Laboratory, Commonwealth Scientific and Industrial Research Organization Livestock Industries,

PO Bag 24, Geelong, VIC 3220, Australia

e-mail: linfa.wang@csiro.au

F. Li

Department of Pharmacology, University of Minnesota Medical School, Minneapolis, MN 55455, USA bat ACE2 molecules. These data suggest that M. daubentoni and $R$. sinicus are likely to be susceptible to SARS-CoV and may be candidates as the natural host of the SARS-CoV progenitor viruses. Furthermore, our current study also demonstrates that the genetic diversity of ACE2 among bats is greater than that observed among known SARS-CoV susceptible mammals, highlighting the possibility that there are many more uncharacterized bat species that can act as a reservoir of SARS-CoV or its progenitor viruses. This calls for continuation and expansion of field surveillance studies among different bat populations to eventually identify the true natural reservoir of SARS-CoV.

\section{Introduction}

Severe acute respiratory syndrome coronavirus (SARS$\mathrm{CoV}$ ) is the aetiological agent responsible for the SARS outbreaks during 2002-2003, which had a huge global impact on public health, travel and the world economy [4, 11]. The host range of SARS-CoV is largely determined by the specific and high-affinity interactions between a defined receptor-binding domain (RBD) on the SARS-CoV spike protein and its host receptor, angiontensin-converting enzyme 2 (ACE2) $[6,7,9]$. It has been hypothesized that SARS-CoV was harbored in its natural reservoir, bats, and was transmitted directly or indirectly from bats to palm civets and then to humans [10]. However, although the genetically related SARS-like coronavirus (SL-CoV) has been identified in horseshoe bats of the genus Rhinolophus $[5,8,12,18]$, its spike protein was not able to use the human ACE2 (hACE2) protein as a receptor [13]. Close examination of the crystal structure of human SARS-CoV RBD complexed with hACE2 suggests that truncations in the receptor-binding motif (RBM) region of SL-CoV spike 
protein abolish its hACE2-binding ability [7, 10], and hence the SL-CoV found recently in horseshoe bats is unlikely to be the direct ancestor of human SARS-CoV. Also, it has been shown that the human SARS-CoV spike protein and its closely related civet SARS-CoV spike protein were not able to use a horseshoe bat ( $R$. pearsoni) ACE2 as a receptor [13], highlighting a critical missing link in the bat-to-civet/human transmission chain of SARSCoV.

There are at least three plausible scenarios to explain the origin of SARS-CoV. First, some unknown intermediate hosts were responsible for the adaptation and transmission of SARS-CoV from bats to civets or humans. This is the most popular theory of SARS-CoV transmission at the present time [10]. Second, there is an SL-CoV with a very close relationship to the outbreak SARS-CoV strains in a non-bat animal host that is capable of direct transmission from reservoir host to human or civet. Third, ACE2 from yet to be identified bat species may function as an efficient receptor, and these bats could be the direct reservoir of human or civet SARS-CoV. Unraveling which scenario is most likely to have occurred during the 2002-2003 SARS epidemic is critical for our understanding of the dynamics of the outbreak and will play a key role in helping us to prevent future outbreaks. To this end, we have extended our studies to include ACE2 molecules from different bat species and examined their interaction with the human SARS-CoV spike protein. Our results show that there is great genetic diversity among bat ACE2 molecules, especially at the key residues known to be important for interacting with the viral spike protein, and that ACE2s of Myotis daubentoni and Rhinolophus sinicus from Hubei province can support viral entry.

\section{Materials and methods}

\section{Cell lines and antibodies}

HeLa cells were grown in Dulbecco's modified Eagle's medium (DMEM) supplemented with $10 \%$ fetal bovine serum (Gibco, USA). Rabbit polyclonal antibodies against ACE2 of $R$. pearsoni (RpACE2) were generated using $R$. pearsoni ACE2 protein expressed in Escherichia coli at the Wuhan Institute of Virology following standard procedures.

Bat sample collection and identification

Bats were sampled from their natural habitats in Hubei, Guangxi, Guizhou, Henan and Yunnan provinces in China as described previously [8]. Bat identification was initially determined in the field by morphology and later confirmed in the laboratory by sequencing the mitochondrial cytochrome $b$ gene from samples of blood cells or rectal tissue as described previously [1].

\section{Bat ACE2 amplification and cloning}

Total RNA was extracted from bat rectal tissue using TRIzol Reagent (Invitrogen, USA) and treating with RNase-free DNase I at $37^{\circ} \mathrm{C}$ for 30 min. First-strand cDNA was synthesized from total RNA by reverse transcription with random hexamers. Full-length bat ACE2 fragments were amplified using the forward primer bAF2 ( $5^{\prime}$-CTTGG TACCATGTCAGGCTCTTYCTGG- $3^{\prime}$ ) and the reverse primer bAR2 (5'-CCGCTCGAGCTAAAAB[G/T/C]GA V[G/A/C $]$ GTCTGAACATCATC- ${ }^{\prime}$ ). The PCR mixture $(25 \mu \mathrm{L})$ contained $0.5 \mu \mathrm{L}$ cDNA, $1.5 \mathrm{mM} \mathrm{MgCl}_{2}$ and $0.2 \mu \mathrm{M}$ of each primer, and the fragments were amplified using the following parameters: $95^{\circ} \mathrm{C}$ for $5 \mathrm{~min}, 35$ cycles of $94^{\circ} \mathrm{C}$ for $30 \mathrm{~s}, 55^{\circ} \mathrm{C}$ for $45 \mathrm{~s}$ and $68^{\circ} \mathrm{C}$ for $3 \mathrm{~min}$, with a final elongation step at $68^{\circ} \mathrm{C}$ for $10 \mathrm{~min}$. All bat ACE2s were cloned into pCDNA3.1 with $K p n I$ and $X h o I$, and this was verified by sequencing.

\section{Chimeric ACE2 construction}

For samples in which full-length ACE2 amplification was unsuccessful, the N-terminal region (1-1,170 bp) was amplified using the forward primer bAF2 and the reverse primer RMR (5'-TTAGCTCCATTTCTTAGCAGGTAG G-3'). Chimeric ACE2 was constructed by combining the $\mathrm{N}$-terminal region of bat ACE2 with the C-terminal portion of human ACE2 at the unique BamHI site $(1,070$ $1,075 \mathrm{bp})$. The chimera was subsequently cloned into pCDNA3.1 with $K p n I$ and $X h o I$ and sequenced as above.

\section{Construction of bat ACE2 mutants}

ACE2 from $M$. daubentoni was chosen to generate a series of ACE2 mutants using a QuikChange II Site-Directed Mutagenesis Kit (Stratagene, USA). The altered amino acid codon for each mutant is indicated as follows: I27T, $\mathrm{N} 31 \mathrm{~K}, \mathrm{~K} 35 \mathrm{E}$, and H41Y. Mutants were confirmed by sequencing.

\section{Sequence analysis}

All bat ACE2s were submitted to GenBank (EF569964, GQ999931-GQ999938). Sequence alignment was performed using ClustalX version 1.83 [15] and corrected manually. A phylogenetic tree based on amino acid (aa) sequences was constructed using the neighbor-joining (NJ) method in MEGA version 4.1. [14]. 
Analysis of ACE2 expression by western blotting

Lysates of HeLa cells expressing human ACE2 or bat ACE2 were separated on a 4-10\% SDS-PAGE gel, followed by transfer to a polyvinylidene difluoride (PVDF) membrane using a semi-dry protein transfer apparatus (Bio-Rad, USA). The membrane was probed with a rabbit polyclonal antibody against the bat ACE2 protein (1:200) at room temperature for $1 \mathrm{~h}$, followed by incubation with alkaline-phosphatase-conjugated goat anti-rabbit IgG $(1: 1,000)$ (Chemicon, Australia). The probed proteins were visualized using NBT and BCIP color development (Promega, USA).

Pseudotype virus infection assays

An HIV-1-luciferase pseudotype virus carrying the SARS$\mathrm{CoV}$ BJ01 $\mathrm{S}$ protein, HIV/BJ01-S, was prepared as described previously [13]. HeLa cells were seeded onto 96-well plates for $18 \mathrm{~h}$ and then transfected with $0.2 \mu \mathrm{g}$ recombinant plasmid containing bat or human ACE2 using $0.5 \mu \mathrm{L}$ Lipofectamine 2000 (Invitrogen, USA) according to the manufacturer's protocol. At $24 \mathrm{~h}$ post-transfection, $30 \mu \mathrm{L}$ medium containing HIV/BJ01-S was added to each well. At $2-3 \mathrm{~h}$ postinfection, unadsorbed viruses were removed, and fresh medium was added. The infection was monitored by measuring luciferase activity, expressed from the reporter gene carried by the pseudovirus, using a luciferase assay kit (Promega, USA). Cells were lysed at $48 \mathrm{~h}$ postinfection by adding $20 \mu \mathrm{L}$ lysis buffer provided with the kit, and $10 \mu \mathrm{L}$ of the resulting lysates were tested for luciferase activity by the addition of $20 \mu \mathrm{L}$ luciferase substrate in a Turner Designs TD-20/20 luminometer. Each infection experiment was conducted in triplicate, and all experiments were repeated three times.

Live virus infection assays

Live SARS-CoV infection was carried out under BSL4 conditions at the Australian Animal Health Laboratory (AAHL) as described previously [16, 17]. Briefly, $48 \mathrm{~h}$ after transfection, the time at which expression of the ACE2 receptor on the HeLa cell surface is optimal, $2 \times 10^{6} \mathrm{TCID}_{50}$ of virus was added to the cells for infection. The cells were fixed $24 \mathrm{~h}$ later by treatment with $100 \%$ methanol for $10 \mathrm{~min}$ and washed five times with PBST. The primary antibody, chicken anti-SARS-CoV S (produced against the recombinant $\mathrm{S}$ protein expressed in E. coli at AAHL), at a 1:500 dilution in 1\% BSA/PBS, was added and incubated with the cells for $1 \mathrm{~h}$ at room temperature. An FITC anti-chicken conjugate (Chemicon, Australia) at $1: 1,000$ in $1 \%$ BSA/PBS was added after washing the cells five times and incubated with the cells for
$1 \mathrm{~h}$. Infection was monitored by immunofluorescent microscopic analysis.

\section{Results and discussion}

Cloning and expression of ACE2 genes from different bat species

ACE2 genes from seven bat species were amplified and cloned (Fig. 1, sFig. 1). Full-length genes were obtained from Rhinolophus ferrumequinum from Hubei province (Rf-HB), R. macrotis from Hubei province (Rm-HB), R. pearsoni from Guangxi (Rp-GX), R. pusillus from Hubei province (Rpu-HB), R. sinicus from Guangxi province (Rs-GX) and R. sinicus from Hubei province (Rs-HB). For the following bat species, amplification of the full-length coding region was not successful, and instead,the N-terminal region was cloned in frame with the $\mathrm{C}$-terminal region of the human ACE2 gene to form a chimeric fulllength ACE molecule: $R$. pearsoni from Guizhou province (Rp-GZ), Myotis daubentonii bat from Yunnan province (Md-YN) and Hipposideros pratti bat from Henan province (Hp-HN). The full-length sequences of bat ACE2 are identical in size to that of hACE2 (805 aa in total). Sequence comparison showed that bat ACE2s are closely related to ACE2s of other mammals and have an aa sequence identity of $80-82 \%$ to human and civet ACE2. The aa identity of ACE2 from different bat families ranges from 78 to $84 \%$, and within the genus Rhinolophus, the sequence identity increases to $89-98 \%$. The major sequence variation among bat ACE2s is located in the N-terminal region, which has been identified in structural studies as the SARS-CoV-binding region [6, 7]. A phylogenetic tree was constructed based on the sequences of bat ACE2 (sFig. 2) using the MEGA package [14].

All ACE2 genes were cloned into a eukaryotic expression vector and used to transfect HeLa cells. Western blot analysis showed that all ACE2s were expressed efficiently and at very similar levels and were recognized by a rabbit anti-bat ACE2 antibody with an apparent molecular weight of approximately 100-130 kDa (Fig. 2c).

Functionality of bat ACE2 as an SARS-CoV entry receptor

To examine the susceptibility of different bat ACE2 molecules to SARs-CoV entry, the HIV/BJ01-S pseudovirus system was used to infect HeLa cells transiently expressing bat ACE2 or human ACE2 genes. Among the bat ACE2s, only MdACE2 (MdACE2) and Rs-HB ACE2 demonstrated significant pseudovirus infection, as deduced from the significantly higher level of luciferase activity in 
Fig. 1 Sequence alignment of SARS-CoV binding regions of ACE2s from 9 bats, civet and human. The GenBank accession numbers of bat, civet and human ACE2 are as follows: human (NM021804), civet (AY881174), Rf-HB

(GQ999931),

Rm-HB (GQ999932), Rs-GX

(GQ999933), Rp-GX

(EF569964), Hp-HN

(GQ999934),

Rp-GZ (GQ999935), Rs-HB

(GQ999936), Md-

YN(GQ999937) and Rpu-HB

(GQ999938). The alignment

was generated using ClustalX

v1.83. In black are single, fully conserved residues. In gray are strongly conserved residues. In light gray are weakly conserved residues. Asterisks indicate residues that interact directly with the receptor-binding domain of the SARS-CoV S protein

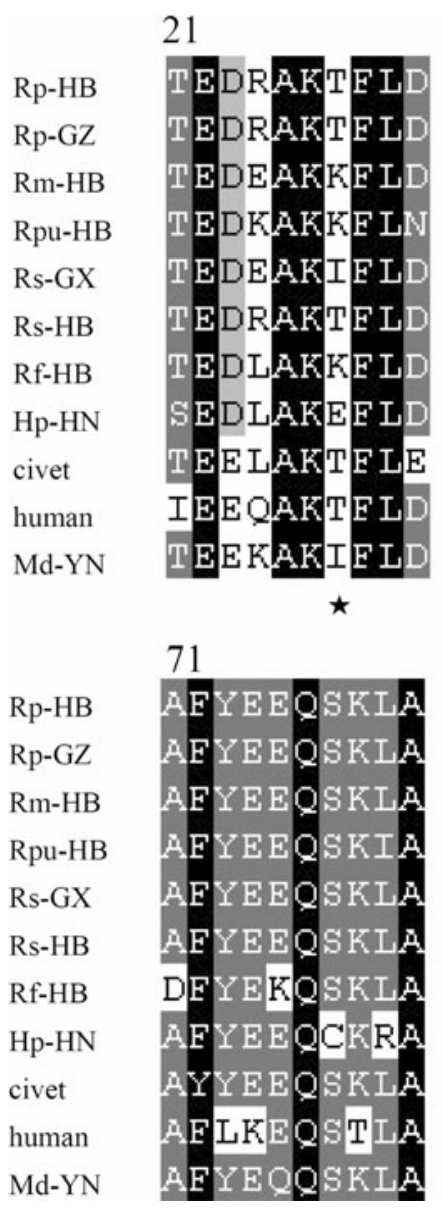

31

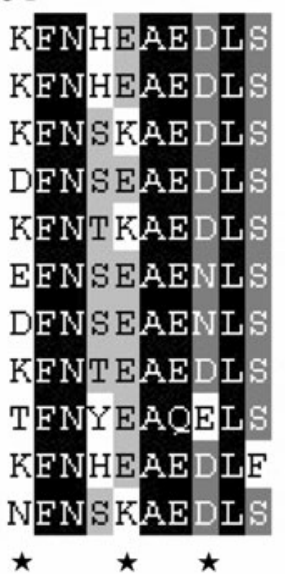

81

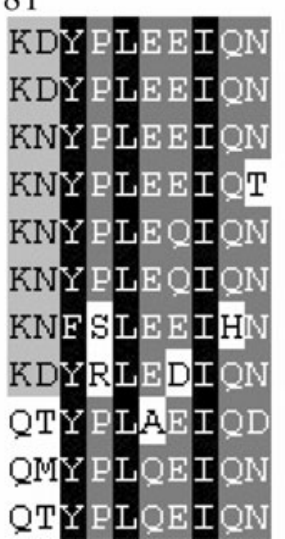

41
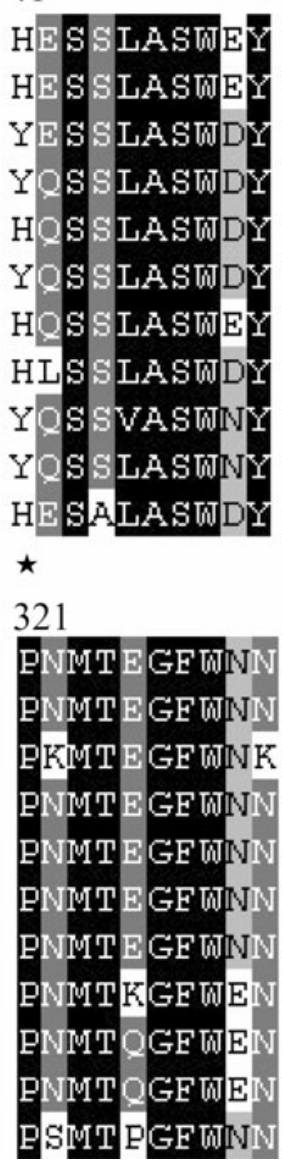

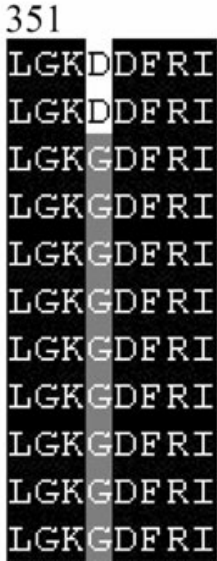

comparison to background activity in the negative control (Fig. 2a). Although such assays are not to be viewed as an absolute quantification of receptor activity, it is nevertheless worth noting that MdACE2-mediated infection seemed to be more efficient than with Rs-HB ACE2. In the same context, it is clear that the bat ACE2s were less efficient overall than the human ACE2 in this particular assay system. The biological significance of this observation remains to be determined. The functionality of MdACE2 and Rs-HB ACE2 as SARS-CoV entry receptors was further confirmed by infection with live virus. As shown in Fig. 2b, both bat ACE2 proteins could clearly support SARs-CoV infection. No attempt was made to quantify infection efficiency in this study due to difficulties encountered in conducting experiments under BSL4 conditions.

\section{Structural modeling of bat ACE2 molecules}

Homologous structural modeling of human SARS-CoV RBD complexed with MdACE2 supports MdACE2 as a receptor for human SARS-CoV $S$ protein. The crystal structure of human SARS-CoV RBD complexed with hACE2 shows that two salt bridges at the SARS-CoV-
hACE2 interface, between hACE2 Lys31 and Glu35 and between hACE2 Lys353 and hACE2 Glu38, are both buried in a hydrophobic environment and contribute critically to the SARS-CoV-hACE2 interactions (Fig. 3a, c) [7]. Disturbance of the formation of either of these salt bridges weakens SARS-CoV-hACE2 binding. The Lys31Glu35 salt bridge at the SARS-CoV-hACE2 interface becomes an Asn31-Lys35 hydrogen bond at the SARSCoV-Md-YNACE2 interface (Fig. 3b), which possibly weakens virus-receptor binding but still is largely compatible with the virus-receptor interface. Thr27 on hACE2 supports the Lys31-Gu35 salt bridge through hydrophobic interactions with Tyr475 (Fig. 3a); Ile27 on MdACE2 supports the Asn31-Lys35 hydrogen bond more efficiently than Thr27 through tighter hydrophobic interactions with Tyr475 (Fig. 3b). Moreover, Tyr41 on hACE2 supports the Lys353-Glu38 salt bridge (Fig. 3c); His41 on MdACE2 supports the same salt bridge less efficiently than Tyr41 (Fig. 3d). Overall, MdACE2 is an efficient receptor for SARS-CoV, despite the fact that its receptor activity is lower than that of hACE2.

Compared with MdACE2, Rs-HB ACE2 contains Glu31 and Glu35, which are not compatible with each other due to their same negative charges, which disfavor 


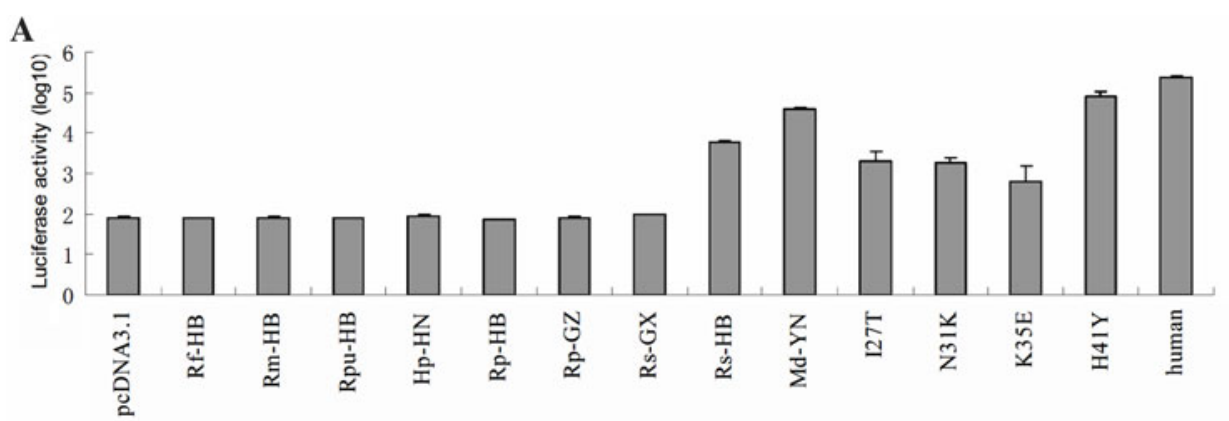

B
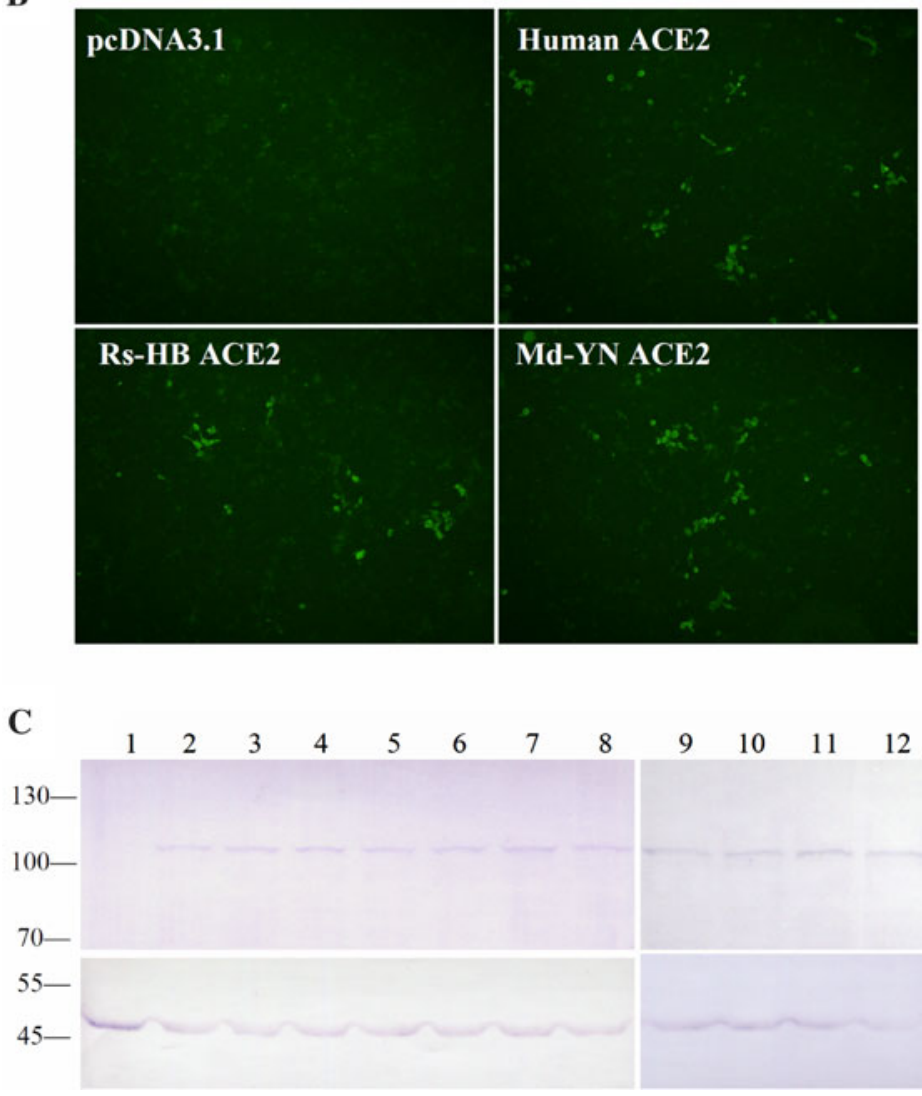

Fig. 2 Testing of the ability of bat ACE2 proteins to mediate pseudovirus HIV/BJ01-S and live SARS- CoV infection. a HeLa cells transfected with plasmids encoding bat and human ACE2s were infected with pseudovirus HIV/BJ01-S. Infectivity was determined by measuring the activity of reporter luciferase as described in "Materials and methods". HeLa cells transfected with pcDNA3.1 and human ACE2 were used as the negative and positive controls, respectively. All tests were performed in triplicate, and the experiments were repeated three times. The error bar represents the calculated standard deviation. I27T, N31K, K35E, and H41Y are mutants of MdACE2 that were made using a QuikChange II SiteDirected Mutagenesis Kit. b SARS-CoV live virus infection using the

virus-receptor binding. However, Rs-HB ACE2 also contains Thr27 and Tyr41, both of which support SARS-CoV entry by contributing favorably to the hydrophobic interactions at the virus-receptor interface. Thus, Rs-HB is a low-efficiency receptor for SARS-CoV. All of the other bat
ACE2s from bats as described in "Materials and methods". HeLa cells transfected with pcDNA3.1 and human ACE2 were used as the negative and positive controls, respectively. c Expression of bat or human ACE2. Lysates from HeLa cells transfected with plasmid expressing human or bat ACE2 were analyzed by western blot. Rabbit anti-bat ACE2 polyclonal antibody (upper panel) or $\beta$-actin monoclonal antibody (lower panel) was used as the primary antibody. Lane 1 vector pcDNA3.1 control; lanes $2-10$ bat ACE2 from samples Rf-HB, Rm-HB, Rpu-HB, Hp-HN, Rp-HB, Rp-GZ, Rs-GX, Rs-HB and Md-YN; lanes 11-14 Md-YN ACE2 mutant I27T, N31K, K35E and H41Y; lane 15 human ACE2. The abbreviations of bat species are given in the main text

ACE2 molecules contain combinations of the aforementioned key residues that are completely incompatible with virus-receptor interactions. More specifically, they either contain same-charged residues at the 31 and 35 positions, which repel each other, or contain His41 and Lys27, which 
Fig. 3 Homologous structural modeling of SARS-CoV and Md-YN ACE2 (MdACE2) interactions. a Critical salt bridge between hACE2 Lys31 and Glu35 and the hydrophobic residues surrounding it, based on the experimentally determined crystal structure of SARS-CoV RBD complexed with hACE2 (PDB 2AJF).

b Homologous structural modeling of the hydrogen bond between MdACE2 Asn31 and Lys35. The modeling was done in the program $\mathrm{O}$ [3]. c Critical salt bridge between hACE2 Lys353 and Glu38 and the hydrophobic residues surrounding it, based on the structure of SARS-CoV RBD complexed with hACE2. d Homologous structural modeling of the salt bridge between MdaACE2 Lys353 and SARS-CoV Glu38 and the hydrophobic residues surrounding it. Structural illustrations were prepared using the program Povscript [2]
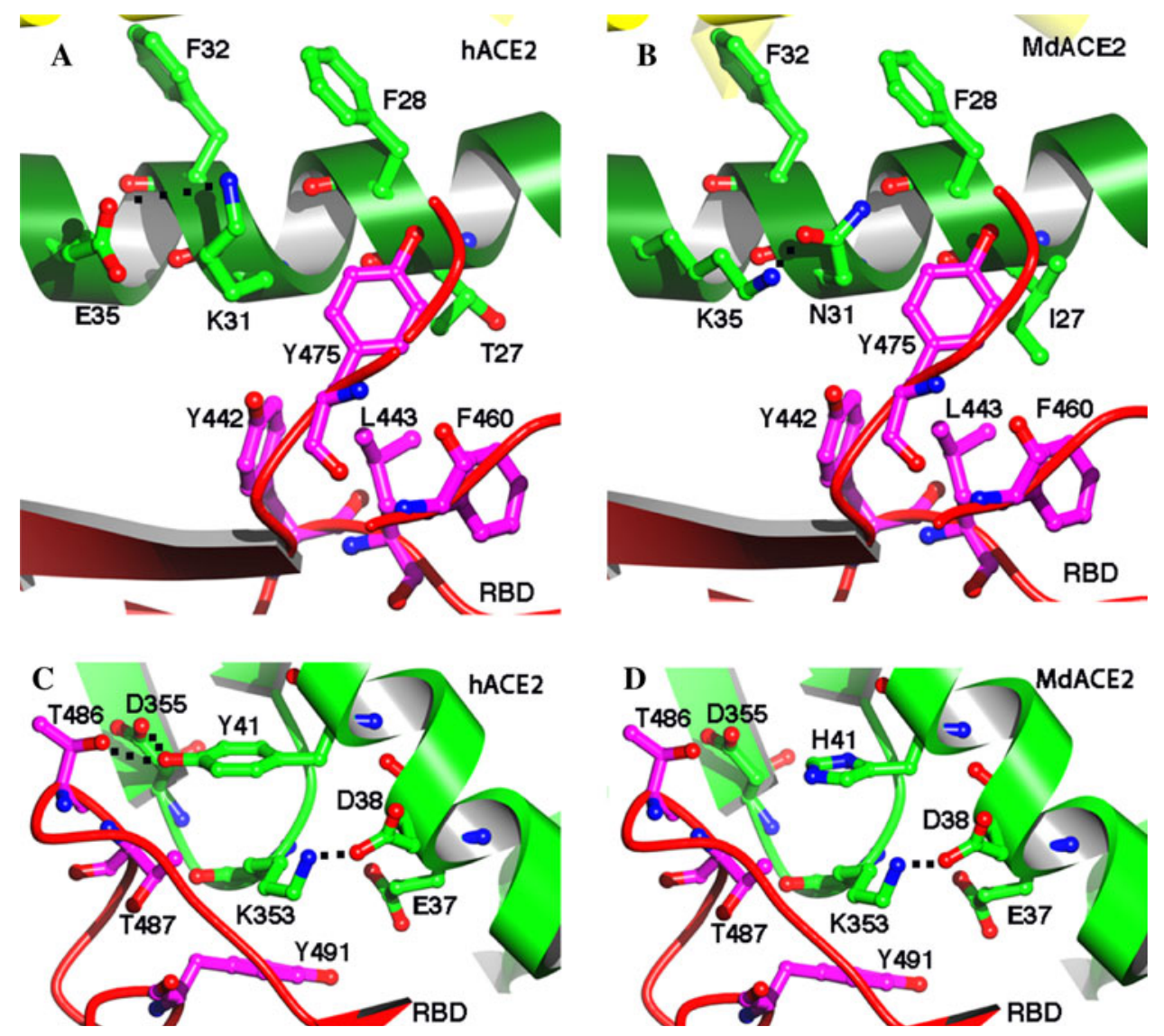

disfavor SARS-CoV binding (Fig. 1). In particular, Lys27 on some of these bat ACE2 molecules is incompatible with certain hydrophobic residues, such as Leu443 and Phe460, on SARS-CoV RBD (Fig. 3a, b). Therefore, these bat ACE2 molecules are not receptors for SARS-CoV.

\section{Site-directed mutagenesis analysis}

To confirm the above homologous structural analysis, we carried out site-directed mutagenesis on MdACE2. Our results show that mutations E31K, K35E, and $\mathrm{I} 27 \mathrm{~T}$ all dramatically decrease the receptor activity of MdACE2, whereas mutation $\mathrm{H} 41 \mathrm{Y}$ greatly increases its receptor activity (Fig. 2a). Therefore, our mutagenesis data further confirmed that key residues in ACE2 determine the receptor activity of MdACE2.

Our finding that $M$. daubentoni and $R$. sinicus could support SARS-CoV infection has important implications in relation to the origin of SARS-CoV. Since all lines of investigation have indicated that ACE2-binding affinity is among the important determinants for SARS-CoV host range, our data would suggest that $M$. Daubentonii and $R$. sinicus have the potential to serve as the direct reservoirs for human SARS-CoV or its highly related civet SARS-
CoV. To further investigate the potential of M. Daubentonii and $R$. sinicus as reservoirs for SARS-CoV, more efforts will have to be directed toward widening the surveillance of bats in these families and in different geographical locations.

Another important finding of our current study is the great genetic diversity of bat ACE2 proteins, which is in contrast to the genetically homogenous hACE2 [10]. Sequence variations of bat ACE2, especially in positions that are critical to SARS-CoV binding, such as residues 27, 31,35 , and 41 , suggest that, in addition to the Md-YN and Rs-HB ACE2s, there may be many other bats with an ACE2 protein that makes them susceptible to SARS-CoV entry. This again highlights the need for more field surveillance and molecular characterization of different bat ACE2 proteins until the true reservoir host of SARS-CoV is identified and its spillover mechanisms and transmission pathways are fully characterized.

Acknowledgments This work was jointly funded by the State Key Program for Basic Research Grants (2005CB523004, 2010CB530100) from the Chinese Ministry of Science, Technology and the Knowledge Innovation Program Key Project administered by the Chinese Academy of Sciences (KSCX1-YW-R-07) to Z.S. and the CSIRO CEO Science Leader Award to L.-F.W. We thank Gary Crameri and Jennifer Barr for help with live virus infection studies. 


\section{References}

1. Cui J, Han N, Streicker D, Li G, Tang X, Shi Z, Hu Z, Zhao G, Fontanet A, Guan Y, Wang L, Jones G, Field HE, Daszak P, Zhang S (2007) Evolutionary relationships between bat coronaviruses and their hosts. Emerg Infect Dis 13:1526-1532

2. Fenn TD, Ringe D, Petsko GA (2003) POVScript+: a program for model and data visualization using persistence of vision raytracing. J Appl Crystallogr 36:944-947

3. Jones TA, Zou JY, Cowan SW, Kjeldgaard M (1991) Improved methods for building protein models in electron density maps and the location of errors in these models. Acta Crystallogr A 47:110-119

4. Ksiazek TG, Erdman D, Goldsmith CS, Zaki SR, Peret T, Emery S, Tong S, Urbani C, Comer JA, Lim W, Rollin PE, Dowell SF, Ling AE, Humphrey CD, Shieh WJ, Guarner J, Paddock CD, Rota P, Fields B, DeRisi J, Yang JY, Cox N, Hughes JM, LeDuc JW, Bellini WJ, Anderson LJ (2003) A novel coronavirus associated with severe acute respiratory syndrome. N Engl J Med 348:1953-1966

5. Lau SK, Woo PC, Li KS, Huang Y, Tsoi HW, Wong BH, Wong SS, Leung SY, Chan KH, Yuen KY (2005) Severe acute respiratory syndrome coronavirus-like virus in Chinese horseshoe bats. Proc Natl Acad Sci USA 102:14040-14045

6. Li F, Li W, Farzan M, Harrison SC (2005) Structure of SARS coronavirus spike receptor-binding domain complexed with receptor. Science 309:1864-1868

7. Li F (2008) Structural analysis of major species barriers between humans and palm civets for severe acute respiratory syndrome coronavirus infections. J Virol 82:6984-6991

8. Li W, Shi Z, Yu M, Ren W, Smith C, Epstein JH, Wang H, Crameri G, Hu Z, Zhang H, Zhang J, McEachern J, Field H, Daszak P, Eaton BT, Zhang S, Wang LF (2005) Bats are natural reservoirs of SARS-like coronaviruses. Science 310:676-679

9. Li W, Zhang C, Sui J, Kuhn JH, Moore MJ, Luo S, Wong SK, Huang IC, Xu K, Vasilieva N, Murakami A, He Y, Marasco WA, Guan Y, Choe H, Farzan M (2005) Receptor and viral determinants of SARS-coronavirus adaptation to human ACE2. EMBO J 24:1634-1643
10. Li W, Wong SK, Li F, Kuhn JH, Huang IC, Choe H, Farzan M (2006) Animal origins of the severe acute respiratory syndrome coronavirus: insight from ACE2-S-protein interactions. J Virol 80:4211-4219

11. Peiris JS, Lai ST, Poon LL, Guan Y, Yam LY (2003) Coronavirus as a possible cause of severe acute respiratory syndrome. Lancet 361:1319-1325

12. Ren W, Li W, Yu M, Hao P, Zhang Y, Zhou P, Zhang S, Zhao G, Zhong Y, Wang S, Wang LF, Shi Z (2006) Full-length genome sequences of two SARS-like coronaviruses in horseshoe bats and genetic variation analysis. J Gen Virol 87:3355-3359

13. Ren W, Qu X, Li W, Han Z, Yu M, Zhou P, Zhang SY, Wang LF, Deng H, Shi Z (2008) Difference in receptor usage between severe acute respiratory syndrome (SARS) coronavirus and SARS-like coronavirus of bat origin. J Virol 82:1899-1907

14. Tamura K, Dudley J, Nei M, Kumar S (2007) MEGA4: molecular evolutionary genetics analysis (MEGA) software version 4.0. Mol Biol Evol 24:1596-1599

15. Thompson JD, Gibson TJ, Plewniak F, Jeanmougin F, Higgins DG (1997) The CLUSTAL_X windows interface: flexible strategies for multiple sequence alignment aided by quality analysis tools. Nucleic Acids Res 25:4876-4882

16. Tu C, Crameri G, Kong X, Chen J, Sun Y, Yu M, Xiang H, Xia X, Liu S, Ren T, Yu Y, Eaton BT, Xuan H, Wang LF (2004) Antibodies to SARS coronavirus in civets. Emerg Infect Dis 10:2244-2248

17. Yu M, Stevens V, Berry JD, Crameri G, McEachern J, Tu C, Shi Z, Liang G, Weingartl H, Cardosa J, Eaton BT, Wang LF (2008) Determination and application of immunodominant regions of SARS coronavirus spike and nucleocapsid proteins recognized by sera from different animal species. J Immunol Methods 331:1-12

18. Yuan J, Hon CC, Li Y, Wang D, Xu G, Zhang H, Zhou P, Poon LL, Lam TT, Leung FC, Shi Z (2010) Intraspecies diversity of SARS-like coronaviruses in Rhinolophus sinicus and its implications for the origin of SARS coronaviruses in humans. J Gen Virol 91:1058-1062 